\title{
Mapping Parallel Programs onto Distributed Computer Systems with Faulty Elements
}

\author{
Mikhail S. Tarkov ${ }^{1}$, Youngsong Mun ${ }^{2}$, Jaeyoung $\mathrm{Choi}^{2}$, and Hyung-Il Choi ${ }^{2}$ \\ ${ }^{1}$ Fault-tolerant computer systems department, Institute of Semiconductor Physics, \\ Siberian branch, Russian Academy of Sciences, \\ 13, Lavrentieva avenue, Novosibirsk, 630090, Russia \\ tarkov@isp.nsc.ru \\ ${ }^{2}$ School of Computing, Soongsil University, \\ 1-1, Sang-do 5 dong, DongJak-gu, Seoul, Korea \\ \{mun, choi, hic\}@ computing.soongsil.ac.kr
}

\begin{abstract}
Mapping one-measured ("line", "ring") and two-measured ("mesh", "torus") parallel program structures onto a distributed computer system (DCS) regular structures ("torus", "two-measured circulant", "hypercube") with faulty elements (processor nodes and links) is investigated. It is shown that: 1) onemeasured program structures mapped better than two-measured structures; 2) when failures are injected to the DCS structure the one-measured structures mapping aggravated very lesser than the two-measured structures mapping. The smaller a node degree of a program graph and the greater a node degree of a DCS graph the better the mapping quality. Thus, the one-measured program structure's mappings are more fault-tolerant than two-measured structure's one and more preferable for organization of computations in the DCS with faulty elements.
\end{abstract}

\section{Introduction}

A Distributed Computer System (DCS) [1-4] is a set of Elementary Machines [EM] that are connected by a network to be program-controlled from these EM. Every EM includes Computing Unit (CU) (a processor with a memory) and a System Device (SD). A functioning of the SD is controlled by the $\mathrm{CU}$ and the SD has input and output poles connected by links to output and input poles of $v$ neighbor EM. A structure of the DCS is described by a graph $G_{s}\left(V_{s}, E_{s}\right), V_{s}$ is a set of EM and $E_{s} \subseteq V_{s} * V_{s}$ is a set of links between EM. For the DCS a graph $G_{p}\left(V_{p}, P_{p}\right)$ of parallel program is determined usually as a set $V_{p}$ of the program branches (virtual elementary machines) that communicate with each other by "point-to-point" principle by transmission of messages across logical (virtual) channels (one- or two-directed) from a set $E_{p} \subseteq V_{p} * V_{p}$. In general case numbers (weights) that characterize computing complexities of branches and intensities of communications between neighbor 
branches are corresponded to nodes $x, y \in V_{p} \quad$ and edges (or arcs) $(x, y) \in E_{p}$ accordingly.

A problem of mapping structure of the parallel program onto structures of DCS is equivalent to the graph isomorphism problem that is NP-complete $[5,6]$. Now efforts of researchers are directed to search effective heuristics suitable for most cases. In many cases observed in practice of parallel programming the weights of all nodes (and all edges) of program graph can be considered equal to each other and can be neglected .In this case a problem of mapping structure of parallel program onto structure of DCS has the following form [5]. The graph $G_{p}\left(V_{p}, P_{p}\right)$ of parallel program is considered as a set $V_{p}$ and a function

$$
G_{p}: V_{p} * V_{p} \rightarrow\{0,1\}
$$

satisfying

$$
G_{p}(x, y)=G_{p}(y, x), G_{p}(x, x)=0
$$

for any $x, y \in V_{p}$. The equation $G_{p}(x, y)=1$ means that there is an edge between nodes $x$ and $y$, that is $(x, y) \in E_{p}$. Analogously the graph $G_{s}=\left(V_{s}, E_{s}\right)$ is determined as a set of nodes (elementary machines (EM)) $V_{s}$ and a function

$$
G_{s}: V_{s} * V_{s} \rightarrow\{0,1\}
$$

Here $E_{s}$ is a set of edges (of links between EM). Suppose that $\left|V_{p}\right|=\left|V_{s}\right|=n$. Let's designate the mapping of parallel program branches to EM by one-to-one function $f_{m}: V_{p} \rightarrow V_{s}$.

The mapping quality can be evaluated by the number of program graph edges coinciding with edges of DCS graph. Let's call this number as a cardinality $\left|f_{m}\right|$ of mapping $f_{m}$ and define it by the expression [5] (the maximum criterion of the mapping quality):

$$
\left|f_{m}\right|=(1 / 2) \sum_{x \in V_{p}, y \in V_{p}} G_{p}(x, y) * G_{s}\left(f_{m}(x), f_{m}(y)\right) \text {. }
$$

The minimum criterion of the mapping quality has the form 


$$
\left|f_{m}\right|=(1 / 2) \sum_{x \in V_{p}, y \in V_{p}} G_{p}(x, y) * L_{s}\left(f_{m}(x), f_{m}(y)\right) .
$$

Here $L_{s}\left(f_{m}(x), f_{m}(y)\right)$ is equal to the distance between nodes $f_{m}(x)$ and $f_{m}(y)$ on the graph $G_{s}$.

\section{Mapping Algorithm}

Let's consider the following approach to the mapping problem [7]. Let initially $f_{m}(x)=x$. Let $e_{p}(x)$ be an environment (a set of neighbors) of a node $x$ on the graph $G_{p}$ and $e_{s}(x)$ be its environment on the graph $G_{s}$. For each node $x \in V_{p}$ we shall test twin exchanges of all nodes $i$ and $j$ if these nodes are satisfied to the condition

$$
\left.i \in e_{p}(x) \& i \notin e_{s}(x) \& j \in e_{s}(x) \& \text { state } j\right)=0,
$$

where state $j)=0$, if a node $j$ has not been exchanged in $e_{s}(x)$ and state $j)=1$ otherwise. Exchanges that don't make worse the performance of mapping $f_{m}$ will be fixed. This approach is based on assumption about high probability of situation when such exchange increasing the number of nodes $i \in e_{p}(x)$ in the environment $e_{s}(x)$ improves (or at least doesn't make worse) the value of performance criterion $\left|f_{m}\right|$. It is obvious that the number of the analyzed exchanges in one evaluation of all nodes $x \in V_{p}$ doesn't exceed the value $v_{p} v_{s} n, n=\left|V_{p}\right|$, where $v_{p}$ and $v_{s}$ are maximum degrees of nodes of graphs $G_{p}$ and $G_{s}$ accordingly. With $v_{p} v_{s}<n$ this approach provides the reduction of computations with respect to the well-known Bokhari algorithm (B-algorithm) [5] and with increase of $n$ the effect of reduction is also increased. On the base of suggested approach the procedure Search has been developed for search of local extremum of function $\left|f_{m}\right|$ in the mapping algorithm (MB-algorithm) that is a modification of the B-algorithm. Besides the MBalgorithm includes following modifications. Firstly initial value of $\left|f_{m}\right|$ is compared with $E_{p}$ and if the equality is carried out then the algorithm is completed. Secondly check-up of the equality $\left|f_{m}\right|=\left|E_{p}\right|$ executed after every completion of the Search. These examinations also lead very often to a cutting down of the algorithm implementation time. 
The MB-algorithm is presented below for the criterion (1). Here: TASK is the description of the graph $G_{p}$, VS is the current mapping $f_{m}$, BEST is the best found mapping $f_{m}, \operatorname{card}(f)$ is the performance criterion of the mapping $f$, card (TASK) is the performance of the graph $G_{p}$ selfmapping, BEST $\leftarrow$ VS is the creation of the copy BEST of the mapping VS, Rand-interchange (VS) is the random exchange of $n$ node pairs in the mapping VS, Output(BEST) is the output of the best mapping $f_{m}$.

Procedure MB;

begin

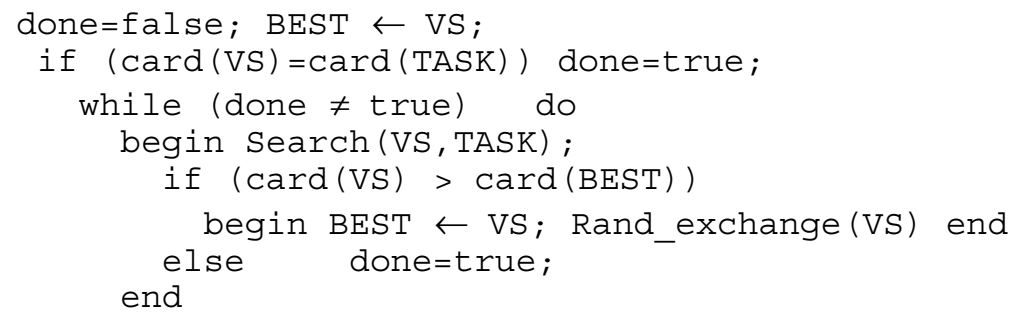

The performance of the MB-algorithm has been investigated [7] on the mapping standard parallel program structures ("line", "ring", "mesh") onto regular graphs of DCS ( $E_{2}$-graph i.e. torus, optimal $D_{2}$-graph i.e. two-measured circulant, hypercube) with a number of nodes $\mathrm{n}$ varying from 8 to 256. Results of MB-algorithm tests show that the cardinality achieved by the algorithm of mapping graph $G_{p}$ onto graph $G_{s}$ with number of nodes $n=\left|V_{p}\right|=\left|V_{s}\right| \leq 256$ is no less than $90 \%$ of the number of nodes in $G_{p}$ for cases when $G_{p}$ is the "line" or the "ring" and no less than $80 \%$ for the case when the $G_{p}$ is the "mesh". The MB-algorithm reduces the time of mapping with respect to B-algorithm in two exponents for mapping one-measured structures ("line", "ring") onto $E_{2^{-}}$and $D_{2}$-graphs and in exponent for mapping two-measured structures ("mesh") onto hypercube. For all that the MB-algorithm doesn't make worse the performance of the mapping with respect to the B-algorithm.

In this paper we investigate the MB-algorithm quality for mapping parallel program structures onto structures of distributed computer systems (DCS) with faulty elements. The mapping quality is investigated for different DCS network topologies (structure types) and for different numbers of faulty processors and links (interconnections).

Usually the DCS graphs initially are regular (torus, hypercube etc) but during the DCS functioning the failures in processors and links can be arised. As a result the parallel program must be remapped onto the nonregular connected functioning part of the DCS. The decentralized operating system of the DCS provides the equality of program branches count to the DCS functioning processor count, i.e. $|V s|=|V p|$ and the computational load is evenly distributed among functioning processors $[3,4]$. In other words, the parallel program can be adjusted to a number of processors in the DCS. 


\section{Conditions for Mapping Parallel Programs onto DCS with Faulty Processors and Links}

How many failures can be tolerant in a DCS for mapping parallel program? There are two types of bounds for the tolerant multiplicity $t$ of failures.

First, a number of faulty nodes must be no more than $(n-1) / 2, n=|V|$. This bound is determined by selfdiagnosability condition [2]. In accordance with the bound, selfdiagnostics algorithm works correctly only when the nonfaulty part of the system has no less than half of the nodes number.

Second, the graph with faulty nodes and edges must be connected. This property is necessary for communications between branches of the parallel program. The probability $p_{t}$ of the DCS graph disconnection and the probability $p_{c}=1-p_{t}$ of the disconnection absence were investigated with respect to the number of faulty nodes and edges. Investigation was realized by Monte Carlo simulation of regular graphs with faulty nodes and edges and the simulation results are presented below.

Figs.1-6 show the simulation plots of $p_{t}$ versus the percentage of failed nodes (Figs.1,3,5) and edges (Figs.2,4,6) for a torus (Figs.1,2), a circulant (Figs.3,4) and hypercube (binary cube) (Figs.5,6) with $n=64$ nodes.

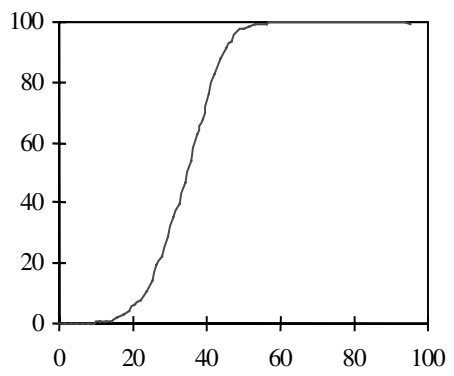

Fig.1

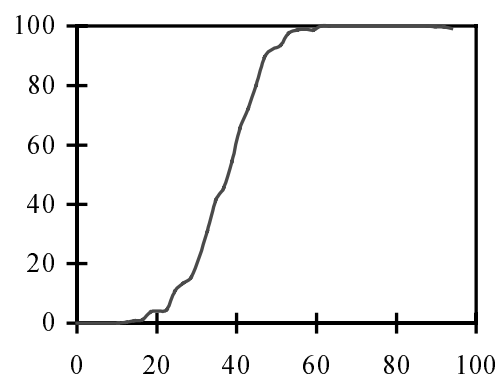

Fig. 3

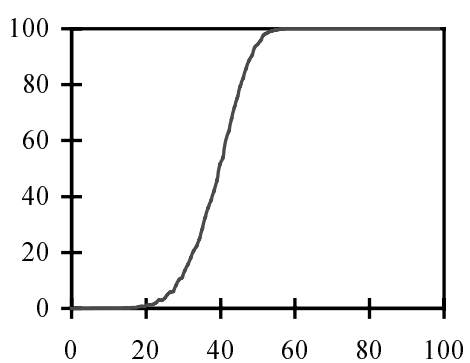

Fig.2

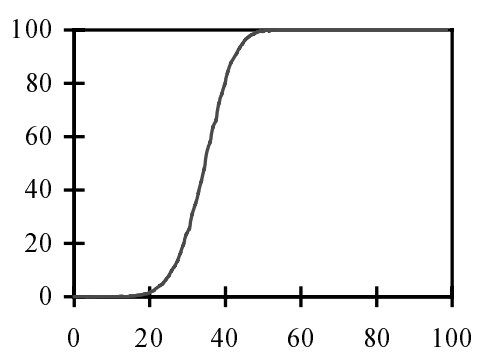

Fig. 4 


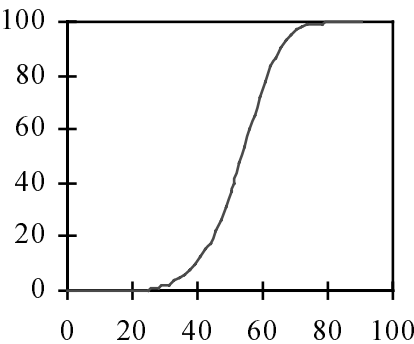

Fig. 5

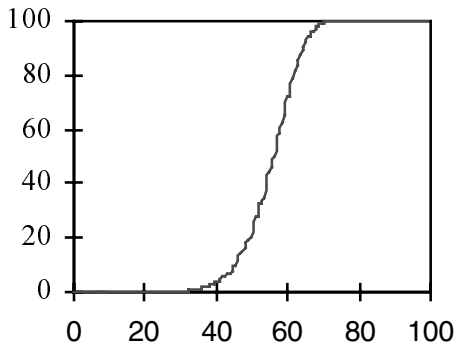

Fig. 6

Simulation results show that:

1)for tori and two-measured circulants $p_{t} \approx 1$ for $t>n / 2$, and for hypercubes $p_{t} \approx 1$ for $t>3 n / 4$

2) for tori and two-measured circulants $p_{c} \approx 1$ for $t \leq 0.2 n$ (see Figs.1-4), and for hypercubes $p_{c} \approx 1$ for $t \leq 0.3 n$ (see Figs.5,6);

These results are true both for graphs with faulty nodes and for graphs with faulty edges. In the second case $n$ is a number of the DCS edges. They show that graph topologies with higher node degree allow the network to sustain a larger number of failures before disconnection. Thus for $t \leq 0.2 n<(n-1) / 2$ all considered topologies are practically connected and we can apply the above mapping algorithm.

\section{Investigation of the Mapping Algorithm}

Four types of parallel programming graphs $G_{p}$ were considered: "line", "ring", "mesh" and "torus". These graphs with the number of nodes from $n=8$ to 256 were mapped onto the DCS structure (torus, two-measured circulant (D2-graph), hypercube) with faulty nodes or faulty edges.

The plan of the mapping algorithm investigation is:

1.Create initial graph $G_{s}$ with a given regular topology and a given number of nodes. 
2. Define a multiplicity of failures $t$ and generate a set of $k$ distorted DCS graphs $G_{s}(i), i=1, \ldots, k$ by means of injection of failures to nodes or to edges of the initial graph.

3. Map the program graph $G_{p}$ onto every distorted DCS graph $G_{s}(i)$ and calculate the mapping quality $\left|f_{m}\right|_{i}$ for every mapping .

4. Calculate average value of relative mapping quality

$$
r_{m}=\frac{1}{k E_{p}} \sum_{i=1}^{k}\left|f_{m}\right|
$$

Results of the mapping algorithm simulation can be distinguished on two groupes:

1. mapping "line" and "ring";

2. mapping "torus" and "mesh".

Table 1. Minimal values $r_{m}(V) / r_{m}(E)$ of average mapping qualities for faulty nodes and for faulty edges

\begin{tabular}{|l|l|l|l|}
\hline$G_{p}$ & Torus & Circulant & Hypercube \\
\hline Line & $0.802 / 0.803$ & $0.801 / 0.802$ & $0.868 / 0.867$ \\
\hline Ring & $0.793 / 0.796$ & $0.797 / 0.790$ & $0.859 / 0.850$ \\
\hline Mesh & $0.571 / 0.792$ & $0.538 / 0.790$ & $0.633 / 0.613$ \\
\hline Torus & $0.500 / 0.595$ & $0.495 / 0.468$ & $0.586 / 0.586$ \\
\hline
\end{tabular}

For the first group (Table.1), if the number of faulty nodes $t<0.2 n$ than the average mapping quality is no less than 0.8 when the DCS graph has topology of torus or circulant and is no less than 0.86 when the DCS graph has topology of hypercube.

For the second group, if the number of faulty nodes $t<0.2 n$ than the average mapping quality is no less than 0.46 when the DCS graph has topology of torus or circulant and is no less than 0.58 when the DCS graph has topology of hypercube.

Figs.7-10 show the simulation plots of the $r_{m}$ versus the number $\mathrm{n}$ of the DCS nodes for several values of the percentage of failed nodes (Tables. 2-5). 
Table 2. Mapping quality, "line $\rightarrow$ torus" mapping

\begin{tabular}{|r|r|r|r|r|r|r|r|}
\hline & 16 & 36 & 64 & 100 & 144 & 169 & 225 \\
\hline $0 \%$ & 1 & 0.971 & 0.905 & 0.909 & 0.923 & 0.929 & 0.938 \\
\hline $10 \%$ & 0.937 & 0.864 & 0.828 & 0.842 & 0.836 & 0.843 & 0.856 \\
\hline $20 \%$ & 0.926 & 0.839 & 0.804 & 0.802 & 0.813 & 0.810 & 0.813 \\
\hline
\end{tabular}

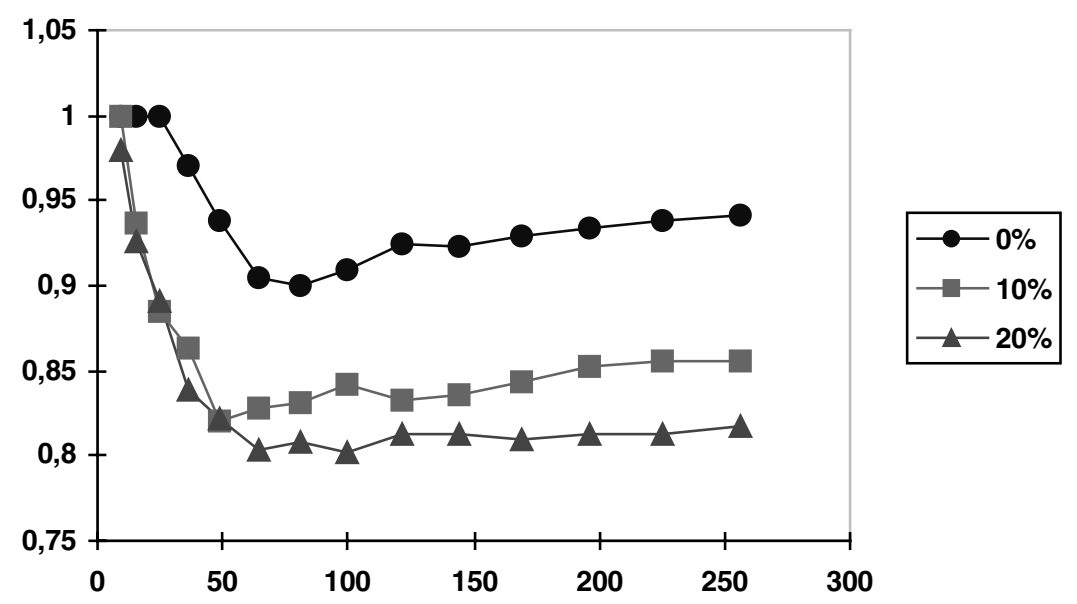

Fig. 7. Mapping quality, "line $\rightarrow$ torus" mapping

Table 3. Mapping quality, "mesh $\rightarrow$ torus" mapping

\begin{tabular}{|r|r|r|r|r|r|r|r|}
\hline & 16 & 36 & 64 & 100 & 144 & 196 & 256 \\
\hline $10 \%$ & 0.865 & 0.698 & 0.674 & 0.639 & 0.633 & 0.641 & 0.628 \\
\hline $20 \%$ & 0.783 & 0.652 & 0.626 & 0.577 & 0.575 & 0.566 & 0.558 \\
\hline
\end{tabular}
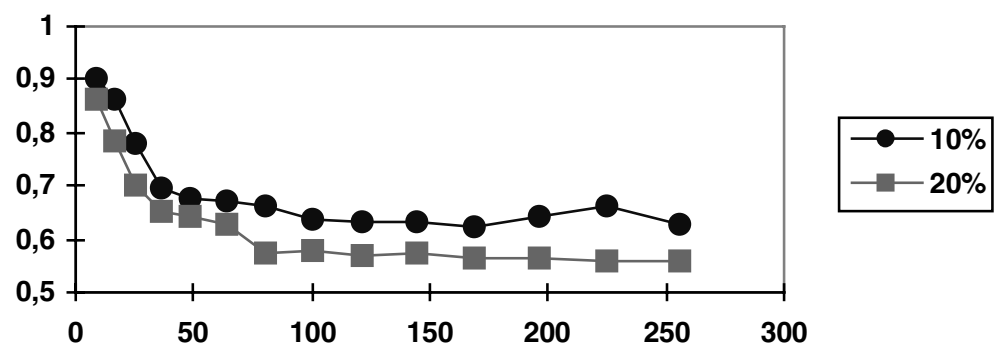

Fig. 8. Mapping quality, "mesh $\rightarrow$ torus" mapping ( $r_{m}=1$ for $0 \%$ percentage of failed nodes) 
Table 4. Mapping quality, "line $\rightarrow$ hypercube" mapping

\begin{tabular}{|c|c|c|c|c|c|c|}
\hline & 8 & 16 & 32 & 64 & 128 & 256 \\
\hline $0 \%$ & 1 & 1 & 1 & 0.968 & 0.937 & 0.937 \\
\hline $10 \%$ & 1 & 0.948 & 0.902 & 0.887 & 0.883 & 0.895 \\
\hline $20 \%$ & 0.919 & 0.909 & 0.880 & 0.868 & 0.870 & 0.878 \\
\hline
\end{tabular}

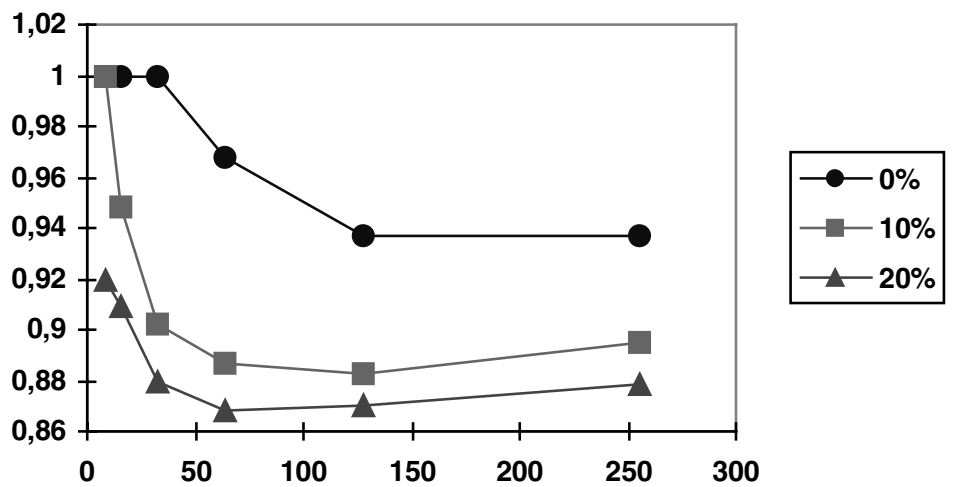

Fig. 9. Mapping quality, "line $\rightarrow$ hypercube" mapping

Table 5. Mapping quality, "mesh $\rightarrow$ hypercube" mapping

\begin{tabular}{|c|c|c|c|c|c|c|}
\hline & 8 & 16 & 32 & 64 & 128 & 256 \\
\hline $0 \%$ & 1 & 1 & 0.923 & 0.812 & 0.776 & 0.771 \\
\hline $10 \%$ & 0.875 & 0.857 & 0.794 & 0.743 & 0.699 & 0.665 \\
\hline $20 \%$ & 0.875 & 0.805 & 0.742 & 0.719 & 0.661 & 0.633 \\
\hline
\end{tabular}

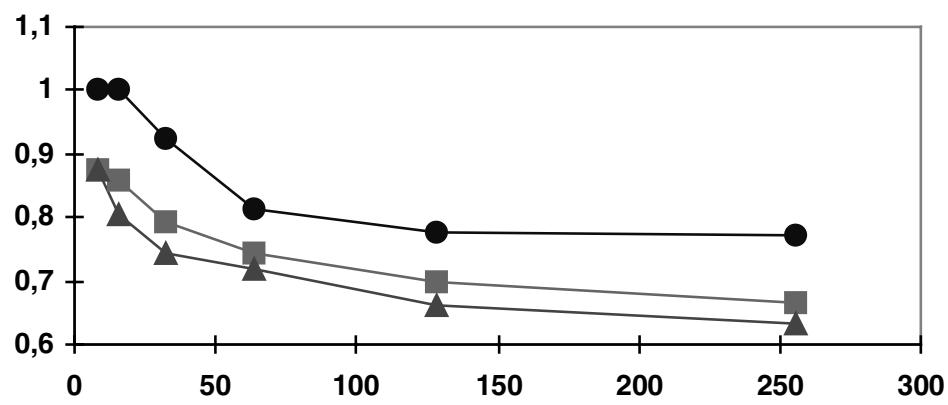

Fig. 10. Mapping quality, "mesh $\rightarrow$ hypercube" mapping 


\section{Conclusion}

Mapping typical graphs ("line","ring","mesh","torus") of parallel programs onto regular graphs ("torus", "two-measured circulant", "hypercube") of distributed computer system (DCS) is considered when the DCS has failures such as faulty processors (nodes) or faulty links (edges). It is shown by the mapping algorithm simulation that:

1) one-measured program structures (line and ring) are mapped better than twomeasured structures (mesh and torus);

2) the one-measured structures mapping is changed to worse very less $(6-15 \%$ for $20 \%$ faulty elements) than the two-measured structures mapping $(15-45 \%)$.

For hypercube the mapping quality values are greater than for torus and twomeasured circulant. The smaller node degree of a program graph and the greater node degree of a DCS graph the greater the mapping quality. Thus, the one-measured program structure's mappings are more fault-tolerant than two-measured structure's one and more preferable for organization of computations in the DCS with faulty elements.

\section{Acknowledgement}

This work was supported by the BK21 program (E-0075).

\section{References}

1. Korneev, V.V.: Architecture of Computer Systems with Programmable Struc ture.Novosibirsk, Nauka (1985)(in Russian).

2. Dimitriev, Yu.K.: Selfdiagnostics of modular computer systems. Novosibirsk, Nauka (1994) (in Russian)

3. Korneev, V.V., Tarkov, M.S.: Operating System of Microcomputer System with Programmable Structure MICROS, Microprocessornie sredstva i sistemy (Microprocessor means and systems). 4 (1988) 41-44 (in Russian)

4. Tarkov, M.S.:Parallel Fault Tolerant Image Processing in Transputer System MICROS-T. Nauchnoe priborostroenie. Vol.5. 3-4 (1995) 74-80 (in Russian)

5. Bokhari, S.H.: On the Mapping Problem, IEEE Trans. Comput., C-30(3), (1981) 207-214

6. Lee, S.-Y., Aggarval, J.K.:A Mapping Strategy for Parallel Processing, IEEE Trans. Comput., C-36(4), (1987) 433-442

7. Tarkov, M.S.: Mapping Parallel Programs Onto Distributed Robust Computer Systems. Proceed. of the 15th IMACS World Congress on Scientific Computation, Modelling and Applied Mathematics, Berlin, August 1997. V.6. Application in Modelling and Simulation. Proc. Ed. By Achim Sydow. (1997) 365-370 